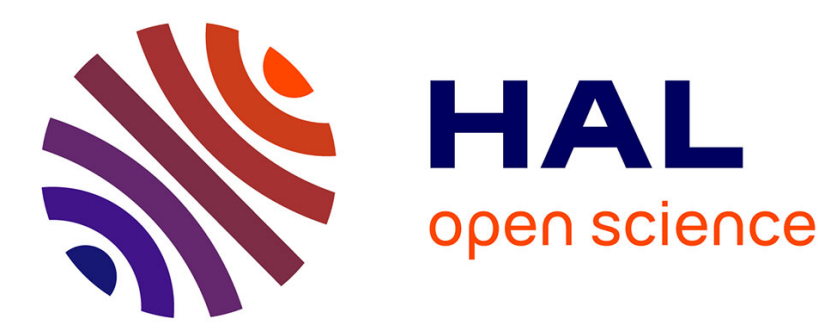

\title{
Leveraging the Power of the Crowd and Offloading Urban IoT Networks to Extend their Lifetime
}

\author{
Géraldine Texier, Valérie Issarny
}

\section{To cite this version:}

Géraldine Texier, Valérie Issarny. Leveraging the Power of the Crowd and Offloading Urban IoT Networks to Extend their Lifetime. LANMAN 2018: IEEE International Symposium on Local and Metropolitan Area Networks, Jun 2018, Washington, DC, United States. pp.1-6, 10.1109/LANMAN.2018.8475050 . hal-01813313

\section{HAL Id: hal-01813313 https://imt-atlantique.hal.science/hal-01813313}

Submitted on 12 Jun 2018

HAL is a multi-disciplinary open access archive for the deposit and dissemination of scientific research documents, whether they are published or not. The documents may come from teaching and research institutions in France or abroad, or from public or private research centers.
L'archive ouverte pluridisciplinaire HAL, est destinée au dépôt et à la diffusion de documents scientifiques de niveau recherche, publiés ou non, émanant des établissements d'enseignement et de recherche français ou étrangers, des laboratoires publics ou privés. 


\title{
Leveraging the Power of the Crowd and Offloading Urban IoT Networks to Extend their Lifetime
}

\author{
Géraldine Texier*†, Valérie Issarny ${ }^{\ddagger}$, \\ $\ddagger$ Inria, France; valerie.issarny@inria.fr \\ †Inria@SiliconValley \\ *IMT Atlantique / IRISA / UBL, France; geraldine.texier@imt-atlantique.fr
}

\begin{abstract}
The widespread deployment of connected Things in our cities allows for the enhanced management of the urban space. However, due to environmental constraints, the connected Things are often battery-powered, which affects the lifetime of the IoT Networks. We believe that the urban IoT networks must leverage the power of the crowd, leading to combine the fixed urban IoT networks with the crowdsensing capabilities of the citizens' smartphones. That is, we integrate the offloading of a portion of the traffic generated by the fixed network to mobile crowdsensing devices that also contribute with relevant urban observations (e.g., for noise pollution monitoring). This paper highlights the key technical features of the resulting solution, whose evaluation shows that we are able to extend the lifetime of the battery-operated IoT network by a up to 7 factor.

Index Terms-IoT, WSN, Routing, Mobile sink, Crowdsensing
\end{abstract}

\section{INTRODUCTION}

Our cities become smarter every day, as they deploy digital services such as intelligent lighting, environmental pollution monitoring, energy and waste management, smart parking, and more. In great part, smart city services rely on Urban Internet of Things (IoT) infrastructures. At a minimum, the connected Things are sensors that perform measurements and send them through the underlying wireless sensor network (WSN) to an Internet server for further analysis. Due to obvious deployment constraints, the Things are often batterypowered, which limits the lifetime of the WSN-based IoT networks. Furthermore, the urban IoT infrastructures primarily serve the long-term monitoring of urban areas, while they are less suited to monitor the citizens' experience. As an alternative, mobile crowdsensing ( $a k a$ mobile phone sensing) is an efficient way to collect a large amount of data about the urban environment and to monitor the citizen's experience. However, the diversity of smartphones and the related heterogeneity of embedded/connected sensors make the correlation of the collected observations more difficult [1]. Following, we believe that the urban IoT infrastructures (i.e., the urban WSNs) and the crowdsensing measurement devices (crowdsensors for short) must work in conjunction so as to both: (1) enhance the quality of the observations about the urban environment, and (2) increase the lifetime of the urban IoT infrastructures. This paper introduces such an approach where we consider that the IoT networks are WSNs implementing the convergecast paradigm (§ II). In a nutshell, we take advantage of the power of the crowd to offload the network traffic so that sensor nodes near the WSN sink do not systematically have to consume significant energy for the routing of the children nodes' traffic (§ III).

The paper specifically contributes with two complementary mechanisms that adapt the routing strategy of the WSN so as to extend the network's lifetime. First, we introduce a load balancing strategy for data forwarding within the WSN, which enhances the fairness of the energy consumption across the WSN nodes. Second, we alleviate the load of the most solicited WSN nodes by offloading a portion of their traffic to nearby related crowdsensors. To validate the proposed mechanisms, we model our problem as a linear program defined as a max-min problem that finds a routing plan to maximize the lifetime of the network considering the expressed constraints (§ IV). The simulation-based evaluation follows, which shows that the proposed offloading onto crowdsensors enables a significant improvement to the network lifetime (§ V). Finally, we conclude with a summary of our contributions and open research questions ( $§ \mathrm{VI})$.

\section{BACKGROUND}

RPL (IPv6 Routing Protocol for Low power and Lossy Networks) [2] is widely deployed to build and maintain the adjacencies of nodes in the WSN. Each RPL node contributes to the creation of a Destination-Oriented Directed Acyclic Graph (DODAG) in a distributed manner. The DODAG interconnects the WSN nodes so that each node sets its preferred parent for forwarding its messages toward the WSN sink that connects the WSN to the Internet. Then, the data is forwarded across the DODAG in a convergecast manner through the shortest path with respect to an RPL objective function taking one or several metrics into account (e.g., hop count or ETX). Therefore, ignoring the background control traffic and the sensing task, a WSN node will spend its lifetime: sending its own data and relaying traffic toward its preferred parent. One way to delay the energy depletion of the WSN due to the convergecast gathering is to introduce extra sinks that are able to absorb and send a portion of the traffic through their own Internet connection. Indeed, transmitting data to an additional sink does not increase the energy consumed by the sender. Rather, it 
spares the energy of the parent node by decreasing the volume of data that the parent would relay otherwise, and so on till the WSN sink. Hence, a well-chosen additional sink can reduce the energy consumption that is due to communication in the WSN and thereby increase the network lifetime.

Minimizing the energy consumption of routing protocols in WSN has been the focus of tremendous work (e.g., see [3]). Several work consider the optimization of RPL for WSN with respect to energy consumption. Existing solutions include: leveraging a metric that represents the nodes' available energy and minimizing the average energy consumption by selecting the paths with the highest energy budget [4]. However, the impact of a very dynamic metric on the DODAG management is not negligible and also creates high routing instability due to frequent changes of the preferred parents.

Another way to reduce the energy consumption due to the routing of data within the WSN is to introduce mobile sinks that take in charge the forwarding tasks over the sensor nodes. Several papers leverage delay tolerant network (DTN) techniques to implement data collection with mobile sinks. The techniques differ according to the constraints that are set regarding the mobility and availability of the sinks (e.g., [5], [6]).

In a way similar to DTN-based solutions, our work leverages mobile sink nodes to reduce the energy consumption of sensor nodes due to message forwarding. However, instead of introducing dedicated sink nodes, we consider the collaboration of the WSN-based urban IoT infrastructures with related urban crowdsensing apps that cities increasingly promote and citizens adopt for the sake of civic engagement. The users' smartphones then relay a portion of the measurements sensed by the infrastructure nodes, which reduces the load of the urban IoT networks and thereby extend their lifetime.

\section{THE SYMBIOSIS OF THE IOT INFRASTRUCTURE AND CROWDSENSING}

Crowdsensing is a powerful mechanism to monitor both the individual and collective exposure of people to urban physical phenomena. However, the large diversity of the sensing devices challenges the accuracy of the contributed observations [1]. The combination of the observations from the fixed urban IoT infrastructure and the crowdsensing devices then contributes to a more accurate knowledge about the urban physical phenomena: the former brings higher accuracy, the latter brings individualized knowledge.

The combination of the IoT infrastructure and crowdsensing devices can further serve extending the WSN lifetime as the smartphones may offer to offload a portion of the infrastructure's traffic. That is, any WSN node may send traffic to a nearby "collaborating" smartphone, so that the smartphone will transmit the data to the server through its own Internet connection. In [7], the authors use smartphones as opportunistic proxies to connect isolated sensors (without Internet connection) to the IoT. In our case, the sensors are not isolated; rather, the smartphone takes over part of the workload of the IoT infrastructure by relaying a portion of its traffic to increase the infrastructure's lifetime.

Although connecting any node with a mobile (smartphone) sink has a cost, sending some traffic to such an extra sink can be very cost-effective when the routing path toward the WSN root is long. The mechanism not only reduces the overall network load, avoiding congestion and contention, but it also increases the lifetime of the fixed IoT network by reducing receptions and emissions at every intermediate node. To validate our claim, this paper provides an evaluation of such an offloading mechanism. In the following, we refer to the smartphones willing to offer a forwarding service to the WSN as mobile sinks or mobile nodes. A sensor can still send data to its parent through the convergecast gathering. However, any sensor able to reach one of the mobile sinks may choose to use it as its next hop to directly send data to the server, so as to reduce the network's energy consumption and thereby delay the network's energy shortage.

As an illustration, we consider the use case that is depicted in Figure 1 and inspired from the Ambiciti crowdsensing app (http://ambiciti.io/) with which users contribute to the monitoring of the individual and collective exposure to the urban noise pollution. The app performs noise measurements and sends them out to a cloud server for data storage and aggregation. Our solution further extends the contribution of the smartphone so that it additionnally offers a communication service to the IoT infrastructure. The smartphone then acts as an additional sink that gathers data from the neighboring sensors, as it moves along the coverage area of the WSN.

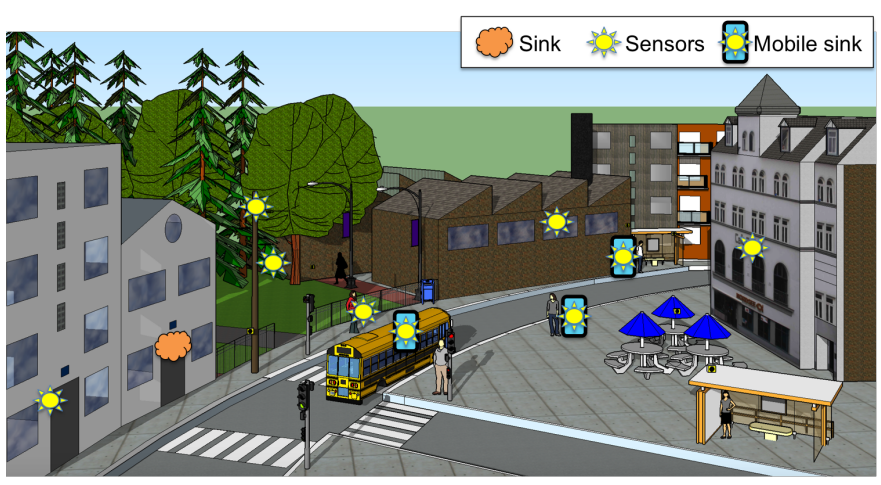

Figure 1: Noise pollution sensing use-case

This paper concentrates on assessing the extent to which offloading the traffic of the IoT infrastructure onto phones that perform related mobile crowdsensing contributes to enhancing the lifetime of the underlying WSN. Still, two complementary technical issues arise: (1) establishing the communication between the infrastructure sensors and the phones, and (2) security.

With respect to the former, current smartphones usually do not implement protocols to communicate with WSNs. Such a feature would require a network interface to connect to nodes in the WSN (e.g., Zigbee, 802.15.4 or BLE). Although except for BLE, most smartphones do not -yet- integrate the 
main interfaces supported by WSN, we consider that this will evolve over time. Further, direct communication between sensors and smartphones is not the only solution. In [8], the authors describe an overhearing mechanism that is able to catch some communication and can then alleviate the global energy consumption. However, these mechanisms require an acknowledgement mechanism to efficiently offload traffic. The fact is that sensors are increasingly able to communicate and we feel that the lack of compatibility is less a technical issue than a market issue since smartphones implementing IEEE Std 802.15.4 have not met their market yet. Therefore, we consider in our study that direct communication between the IoT infrastructure and smartphones is not a blocking point and will be available in the future.

As for the security aspect, we assume use cases where the user downloads the crowdsensing application that is part of the urban sensing system and is able to collect and send data to the dedicated platform. Therefore, the user's device has obtained the required credentials and authorizations to submit data to the server. Our goal is not to develop considerations on security issues already exposed in [9].

\section{WSN OFFLOADING TO CROWDSENSORS}

\section{A. System model}

Our urban sensing system combines two distinct contributors: the urban sensing infrastructure and the crowdsensing app run on the phones of individuals. The sensing infrastructure is a WSN, which we model as a set $\mathcal{N}_{\mathcal{I}}$ of sensors interconnected by a set $\mathcal{E}_{\mathcal{I}}$ of wireless links. The topology of the WSN is represented by a directed graph $\mathcal{G}_{\mathcal{I}}=\left(\mathcal{N}_{\mathcal{I}}, \mathcal{E}_{\mathcal{I}}\right)$. The WSN implements a convergecast protocol toward the WSN sink $s$, and the nodes running the crowdsensing app act as additional, opportunistic mobile sinks.

1) Mobility model: In our system, a crowdsensor may act as a mobile sink if its user/owner is in the communication range of a related WSN sensor and if routing the traffic via that node contributes to the energy efficiency of the WSN.

We more specifically concentrate on the connectivity with the urban users who are pedestrians and/or take city buses. Thus, the crowdsensing devices follow trajectories that are defined either by the bus planning or constrained by the configuration of the city (e.g. they follow the street curb). Pedestrians may change their path but the bus route is fixed. Therefore, we model the mobility of any mobile sink as a location-restricted mobility (LRM) [10]. We identify positions where the smartphone's owner stays possibly long enough to collect data (e.g., while waiting for the bus or for the "Walk" signal at the crosswalk). We consider an arbitrary subset of crowdsensors $\mathcal{M}=\left\{m_{1}, \ldots, m_{n}\right\}$ that act as (potential) mobile sinks when they transit through the identified positions. The parameters associated to our model are then the number of considered mobile sink positions and the duration of their availability.

Given the WSN topology together with the communication radius $r_{m}$ (which we assume identical for all nodes without loss of generality) and the positions of the (potential) mobile sinks, we determine which sensors are able to communicate with a mobile sink and the quantity of data it is able to relay.

The above leads us to define the set $\mathcal{E}$ of links that add the potential adjacencies between sensors and mobile nodes to $\mathcal{E}_{\mathcal{I}}$. Similarly, we integrate the discretized positions of the mobile sinks in the graph $\mathcal{G}_{\mathcal{I}}$, which forms the graph $\mathcal{G}=(\mathcal{N}, \mathcal{E})$ with $\mathcal{N}=\mathcal{N}_{\mathcal{I}} \cup \mathcal{M}$.

Figure 2 illustrates the construction of the graph integrating mobile sinks (right column) to an initial WSN (left column) in which all the parents may forward data.

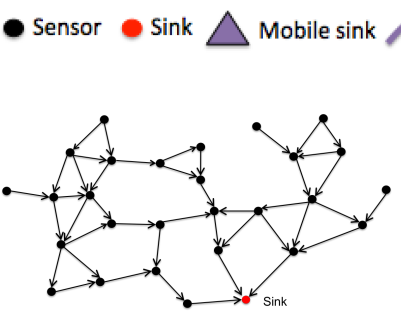

(a) Without mobile node

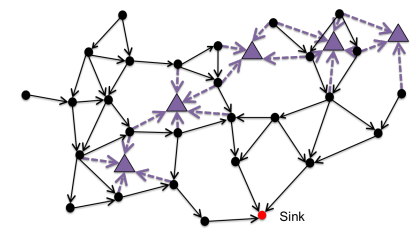

(b) With mobile nodes
Figure 2: Construction of the network topology

We note that the underlying RPL standard provides two strategies to relay communications when several sinks are available. One simple solution, which does not impact the DODAG creation mechanism, nor the way RPL forwards the traffic, is to introduce a virtual node as parent of the sinks. The second solution requires the creation of a different uncoordinated DODAG instance for each sink. Nodes communicate mainly within one DODAG instance to send traffic to the instance's sink. A node can change the sink that will receive a data by transferring it to a different DODAG instance. Several rules restrict the data transfer between DODAG instances to avoid routing loops.

2) Energy model: We choose a simple energy consumption model for (the infrastructure) sensors: each sensor is battery supplied and has an initial energy budget $E$ that is dedicated to communication, and $e(i)$ provides the energy consumption of node $i$. The sink $s$ is power supplied, therefore $e(s)=0(s$ has an infinite energy).

Mobile nodes are smartphones, powered by a rechargeable battery allowing the budget of available energy $E_{m}$. The protocol used to send data from the sensor to the mobile sink varies across sensors; we represent the related cost as a connection cost $e_{c}$.

The reception and the emission of one observation/datum require the same amount of energy, which we denote as $e_{t}$ when the receiver is a sensor, and $e_{m}$ when it is a mobile sink. We denote the energy consumed by a sensor when it does not transmit or receive sensed data as $e_{\text {sleep }}$.

For the sake of simplicity, we consider that a mobile sink has the same transmission capacity for any of its neighbors. Dealing with diverse capacities may be addressed through the integration of an energy cost vector.

3) The network lifetime metric: We characterize the network lifetime as the time between the first activation of the 
network and the time when at least one of the sensors runs out of power. This is the most constrained definition of the network lifetime. The definition may be relaxed in future work by considering that the network remains in operation as long as a given percentage of sensors are still available and/or the/a sink remains reachable. Over its lifetime, a sensor gathers observations and sends them to the application server either through the WSN sink based on the convergecast data gathering, or through one of the mobile sinks. In addition, non-leaf sensors relay data on behalf of their neighbors. We can then express an upper bound for the network lifetime, which is the upper bound of a sensor lifetime, i.e. the time needed for a sensor to run out of energy when sending only its observations and not forwarding any message. An upper bound on the sensor's daily energy consumption is given by $e_{\text {sleep }}+q_{t} * e_{t}$. The upper bound on the sensor lifetime is expressed in days by:

$$
U=\frac{E_{s}}{e_{\text {sleep }}+q_{t} * e_{t}}
$$

Consider for example a sensor based on the widely used TI CC2538 chip. According to the data sheet available at http: //www.ti.com/lit/ds/symlink/cc2538.pdf, the energy consumed during one second in sleep mode (power mode 2 ) is between $1.3 \mu \mathrm{A}$ and $2 \mu \mathrm{A}$, and $50 \mathrm{~mA}$ is an estimation of the energy needed to both wake up and transmit data. We overestimate the maximum lifetime of the sensor when powered with two AA batteries with a capacity of $2500 \mathrm{mAh}$. The maximum sensor lifetime is 5.5 years for a daily traffic of one transmission every second, and 100 years for a daily traffic of one transmission every minute.

\section{B. Routing strategy}

We adopt traffic engineering principles to extend the lifetime of the network, where we combine the following three approaches:

- Load balancing: We take advantage of the mesh network to allow load balancing in the WSN. Unlike basic RPL routing, the traffic is distributed among multiple parents to reduce the energy consumption on the shortest path to a sink, which leads to investigate how a node should divide its outgoing traffic toward its parents. Similarly, we study the amount of data that each node can relay for its children. Note that we do not focus at the time at which the data should be sent in our presentation. Therefore, we concentrate on a flow routing problem that does not take scheduling into account.

- Routing optimization: We compute the percentage of traffic to send to each parent to maximize the network lifetime.

- Introduction of mobile sinks: We take into account the mobile crowdsensing nodes that are in the relevant area and onto which the urban IoT infrastructure may possibly offload traffic. We specifically focus on the amount of data that a mobile node should collect to extend the lifetime of the infrastructure. We further model the motion of any mobile sink by defining a corresponding sink with a limited transmission at every given place where the mobile sink remains long enough.

\section{Computing the routing strategy with a linear program}

We want to compute a routing strategy that determines for each node the percentage of traffic it should forward to each of its neighbors -including a mobile sink if available- in its coverage area in a way that maximizes the network lifetime.

We formulate the problem as a flow routing problem that maximizes the network lifetime. We recall that the lifetime of the network ends as soon as one of the WSN sensors runs out of energy (where we ignore the other cases of sensor failures due to the focus of our work). Thus, we want to increase the lifetime of this specific sensor.

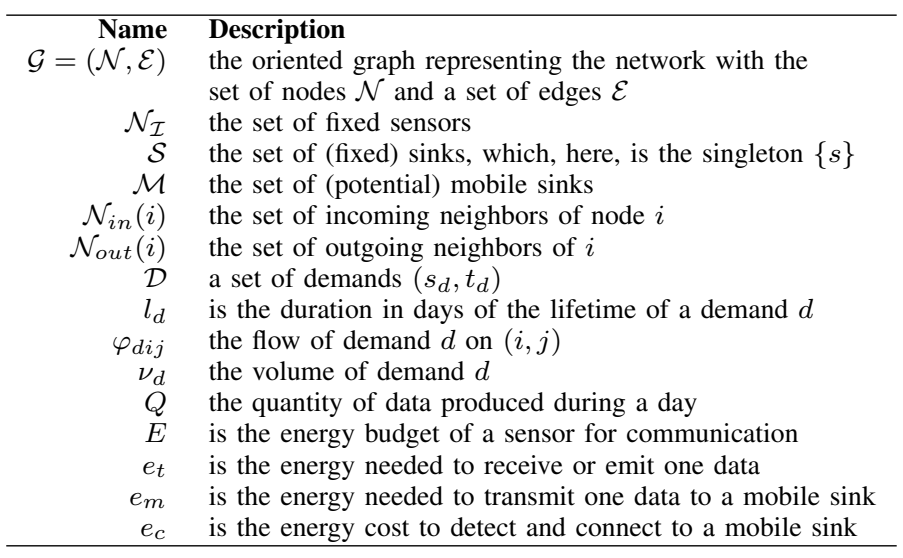

Table I: LP notations

Consider the variables and constants defined in Table I. The above Linear Program -LP- Formulation of our max-min problem finds a routing plan that maximizes the lifetime of the network under the specified constraints:

- The traffic forwarding ensures the flow conservation (1a).

- The traffic exits either at the WSN sink or any of the mobile sinks (1b). 
- The network lifetime is expressed in days (1c).

- A sensor spends its energy budget for communication to send its traffic and relay its neighbors' traffic to its parents or a mobile node (1d).

Solving the LP provides the quantity of flow that each node has to send to its neighbors to ensure that the network has the longest lifetime. We recall that the LP shows how each node shares its traffic toward its ancestors and does not deal with the scheduling of the exchanges. For example, the output of the LP resolution can indicate that a sensor $A$ with three neighbors $B, C$ and $D$ should send $30 \%$ of its traffic to $B, 70 \%$ to $C$ and nothing to $D$ (or $30 \%$ to $B, 20 \%$ to $C$ and $50 \%$ to $D$ ). In the case of routing within the WSN only, the scheduling strategy may be implemented based on that routing plan in a straightforward way. However, the routing toward mobile sinks must account for the actual presence of the nodes. One solution is to send in priority the traffic to the mobile sink -if one is present- until the related traffic quota is reached. If there is no mobile sink that is reachable when the sensor wants to send data, it can either: store it, wait for the next mobile sink, or send it to another neighboring sensor designated as next hop by the routing plan.

Algorithm 1 implements the routing plan where each sensor has access to two data structures describing its adjacencies: (1) $S_{u c c_{m}}$, represents the positions where the sensor can potentially find a mobile sink, (2) $S u c c_{f}$, describes the set of parents -that are not mobile sinks- in the DODAG. An element of $S u c c_{m}$ is described by: the quantity $T T$ of data that it should relay, the quantity $S T$ of data that it has already relayed, and the method ispresent that indicates if a mobile sink is available at this position at the specific moment or not.

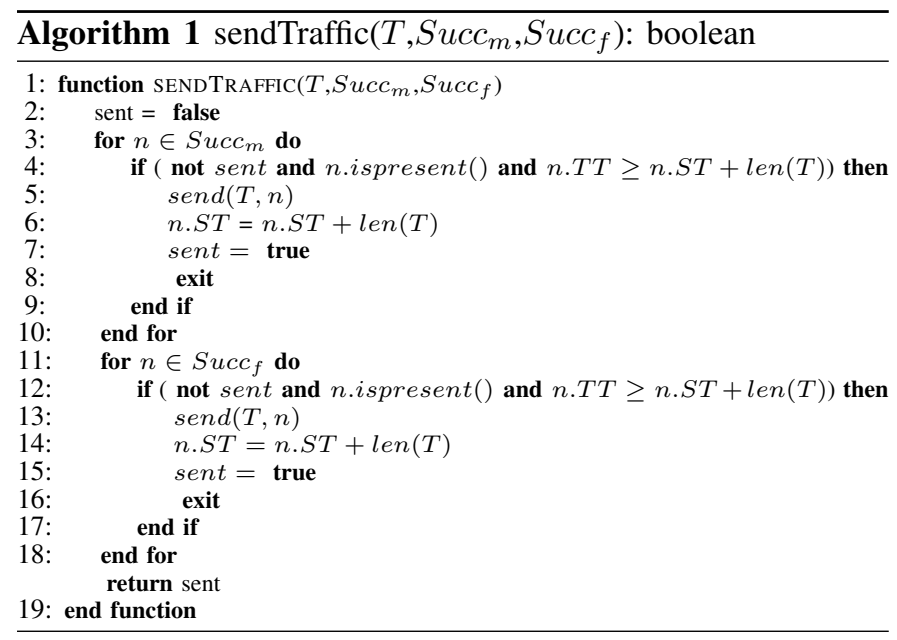

\section{Assessment}

We provide a simulation-based evaluation where we consider a WSN composed of 27 sensors and one sink. We implemented four strategies (see Fig. 3): (1) SPF builds a shortest path tree with the sink as root to simulate the solution implemented by routing protocols such as RPL; (2) LB allows nodes to send traffic to several parents, implementing a load balancing strategy within the WSN; (3) mn5N implements a routing exploiting mobile sinks based on 5 mobile nodes moving along the North path; (4) $\mathbf{m n 5 S}$ is as $\mathbf{m n 5 N}$ but nodes move along the South path.

For each strategy, we consider the following numerical values. The 27 sensors generate a daily traffic of one datum per minute. The sensors are powered by one AA battery with a capacity of $2500 \mathrm{mAh}$. The energy consumed during the sensor sleep mode is set to $e_{\text {sleep }}=2 \mu \mathrm{A}$, while $e_{t}=50 \mathrm{~mA}$ is the energy necessary to transmit data to another node in the network but the transmission to a mobile node involves an extra cost of $e_{m}=100 \mathrm{~mA}$ corresponding to the cost of both the connection to the mobile node, and the overhead of sending data with a different technology.

Figure $3 \mathrm{c}$ provides the LP results for the four aforementioned strategies. SPF highlights the behavior of RPL when data is collected by convergecast. The nodes near the sink transmit a number of packets proportional to the depth of their subtree, leading to a network lifetime that that is less than 5 years. Figure 4a shows the lifetime of each sensor as a function of distance from the sink for the SPF strategy, which depends directly on the lifetime of the direct neighbors of the sink. Indeed, these nodes become short of energy, while about $70 \%$ of the total energy of the network is still available. Figure $3 \mathrm{c}$ shows that the LB strategy improves the network lifespan by $23 \%$ to reach nearly 6 years. The improvement is mainly due to a better involvement of the nodes at a distance 2 from the sink (see Fig. 4b).

Figure $3 \mathrm{c}$ shows that offloading the WSN traffic onto mobile nodes multiplies the network lifetime by 4 (resp. 7) when the mobile nodes are located on the North (resp. South) path, leading to a lifetime of 20 (resp. 33) years. The analysis of the energy consumption of each node as a function of their distance from the WSN sink (see Fig. 4c) confirms a better spread of the nodes contribution in $\mathbf{m n} 5 \mathbf{N}$, including the furthest ones. The results are even more striking on the South path (not shown for space reasons) where nodes from 1 to 5 hop distance from the sink consume $100 \%$ of their energy while nodes at 6 hop distance consume on average 32\% of their energy. The mobile nodes on the south path are located near the nodes furthest away from the sink. They collected data whose relaying would have solicited several nodes and were therefore the most energy-consuming for the network.

In the presented work, we have implemented a simple energy model, considering a uniform transmission cost. In [11], Khan et al. study the energy efficiency of WSN by collecting data through static vs mobile sinks. They show the relationship between the radius of the sink mobility and the energy spent during the data collection. As part of our future work, we will leverage these results and model the energy consumption of nodes as a function of the distance between the node and the mobile sink.

\section{CONCLUSION}

Crowdsensing applications promote the monitoring of urban phenomena through the users' smartphones, including 


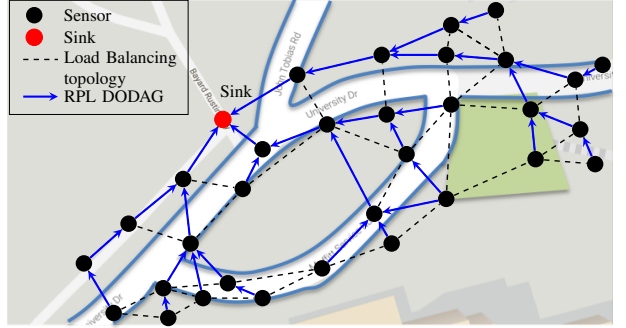

(a) SPF and LB

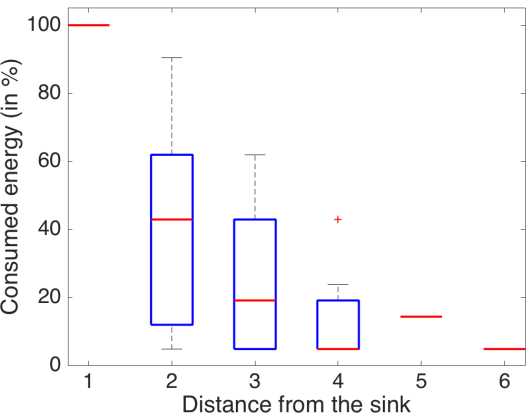

(a) SPF

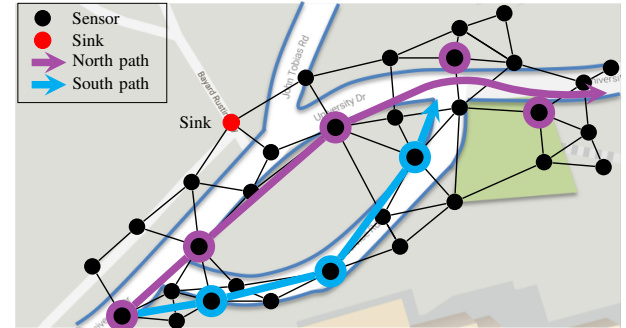

(b) Mobile sinks along North and South paths

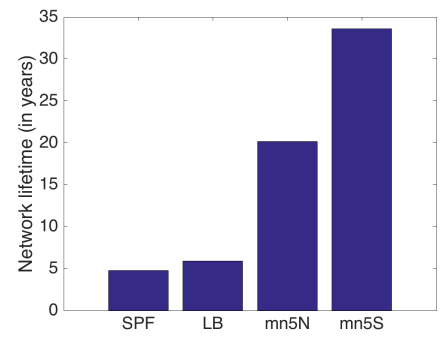

(c) Optimal lifetimes

Figure 3: Evaluated strategies

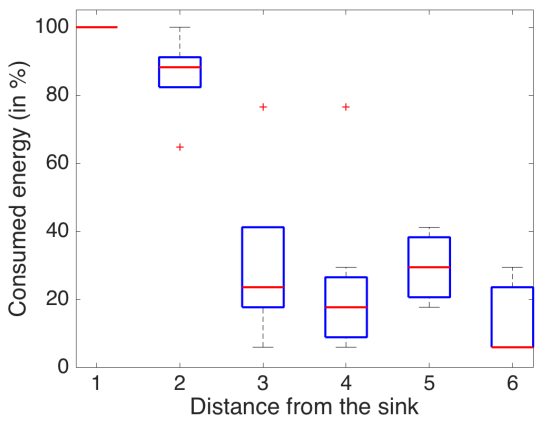

(b) LB

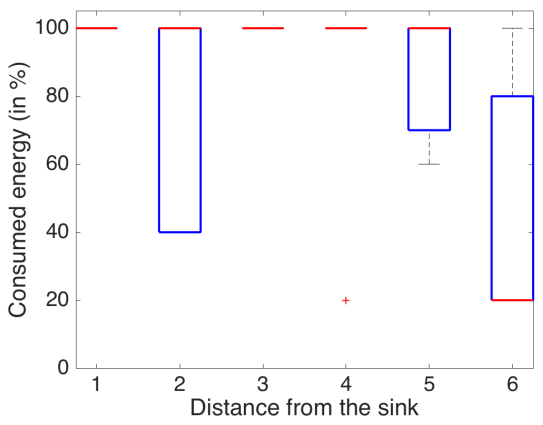

(c) North path

Figure 4: Sensor lifetime analysis

measurements using the connected sensors. Such crowdsensed observations are transmitted by the application to a server using the phone's Internet connection (e.g., 4G or WiFi). However, because of the diversity of the contributing users and devices, the observations are often difficult to correlate and exploit. In the case of physical measurements, the a priori calibration of the sensors and/or correlating the measurements with a fixed IoT infrastructure providing reference measurements serve overcoming this issue. We then argue that urban IoT infrastructures and crowdsensing applications benefit from closer interactions.

We extend the (urban) WSN-based IoT infrastructure with mobile sinks that run related crowdsensing apps and are capable of receiving and transferring traffic to a server with their own Internet connection (e.g., 4G). We compute the supporting routing plan using a LP formulation that maximizes the WSN lifetime. Our simulation-based evaluation shows that our strategy would considerably extend the network lifetime. Our work then opens up several research issues that we investigate as part of our ongoing and future work. One such issue relates to the integrated load balancing strategy that requires transforming the traditional routing mechanism based on the next hop information into a table with proportional routing. A node's proportional routing table should indicate the percentage of traffic it should send for each of its parent. Although the table will be larger, it may alleviate the impact of temporary failures.

\section{REFERENCES}

[1] V. Issarny, V. Mallet, K. Nguyen, P.-G. Raverdy, F. Rebhi, and R. Ventura, "Dos and Don'ts in Mobile Phone Sensing Middleware: Learning from a Large-Scale Experiment," in ACM/IFIP/USENIX Middleware, 2016.

[2] T. Winter, P. Thubert, A. Brandt, J. Hui, R. Kelsey, P. Levis, K. Pister, R. Struik, J. Vasseur, and A. R., "RPL: IPv6 Routing Protocol for LowPower and Lossy Networks," IETF, RFC 6550, 2012.

[3] N. A. Pantazis, S. A. Nikolidakis, and D. D. Vergados, "Energyefficient routing protocols in wireless sensor networks: A survey," IEEE Communications surveys \& tutorials, vol. 15, no. 2, pp. 551-591, 2013.

[4] O. Iova, F. Theoleyre, and T. Noel, "Using multiparent routing in RPL to increase the stability and the lifetime of the network," Ad Hoc Networks, vol. 29, pp. 45-62, 2015.

[5] H. Lee, M. Wicke, B. Kusy, O. Gnawali, and L. Guibas, "Data stashing: energy-efficient information delivery to mobile sinks through trajectory prediction," in 9th ACM/IEEE International Conference on Information Processing in Sensor Networks. ACM, 2010.

[6] S. Batabyal and P. Bhaumik, "Mobility models, traces and impact of mobility on opportunistic routing algorithms: A survey," IEEE Coтmunications Surveys \& Tutorials, vol. 17, no. 3, 2015.

[7] B. Billet and V. Issarny, "Spinel: An opportunistic proxy for connecting sensors to the Internet of Things," ACM Transactions on Internet Technology (TOIT), vol. 17, no. 2, pp. 1-21, 2017.

[8] A. F. Harris III, V. Khanna, G. Tuncay, R. Want, and R. Kravets, "Bluetooth low energy in dense IoT environments," IEEE Communications Magazine, vol. 54, no. 12, pp. 30-36, 2016.

[9] S. Gisdakis, T. Giannetsos, and P. Papadimitratos, "Security, privacy, and incentive provision for mobile crowd sensing systems," IEEE Internet of Things Journal, vol. 3, no. 5, pp. 839-853, 2016.

[10] F. Al-Turjman, M. Karakoc, M. Gunay, and A. Noureldin, "Routing mobile data couriers in smart-cities," in Communications (ICC), 2016 IEEE International Conference on. IEEE, 2016.

[11] M. I. Khan, W. N. Gansterer, and G. Haring, "Static vs. mobile sink: The influence of basic parameters on energy efficiency in wireless sensor networks," Computer communications, vol. 36, no. 9, pp. 965-978, 2013. 